10.2478/v10367-012-0011-y

JAN ŠAFÁŘ, PAUL WILLIAMS

General Lighthouse Authorities of the UK and Ireland

FRANTIŠEK VEJRAŽKA

Czech Technical University, Prague

\title{
ACCURACY PERFORMANCE OF E-LORAN RECEIVERS UNDER CROSS-RATE INTERFERENCE CONDITIONS
}

\begin{abstract}
According to a recent Business Case produced by the General Lighthouse Authorities of the United Kingdom and Ireland (GLAs), e-Loran is the only system that, when combined with GNSS, can achieve cost effective resilient Positioning, Navigation and Timing (PNT) by 2018 for maritime e-Navigation. The GLAs currently operate a trial e-Loran service from Harwich, UK and are working towards establishing e-Loran Initial Operational Capability (IOC) in the seven busiest UK ports and port approaches by mid-2013. A future extension of e-Loran coverage to the entire GLA service area will require the installation of additional transmitting stations. When planning the installation of e-Loran transmitters service providers will need a good understanding of the effects of the new signals on the system's performance. Since all e-Loran stations share the same frequency band and the e-Loran signals propagate over vast distances, special attention needs to be paid to the issue of intra-system interference. This is also referred to as Cross-Rate Interference (CRI) and is inherent to the way e-Loran operates.

In this paper we examine the impact of CRI on the position accuracy performance of e-Loran receivers. First, a signal processing model for a typical e-Loran receiver is developed. This could provide the e-Loran community with a unified framework for receiver performance evaluation. Numerical and, where possible, analytical results obtained from the model are then presented, describing the achievable accuracy performance under different interference conditions. The theoretical results are also compared to those obtained from measurements made on a commercially available receiver driven by a signal simulator.

Our analysis shows that modern e-Loran signal processing algorithms can achieve a substantial reduction of the negative effects of CRI. However, there is still an appreciable residual effect, which should be taken into account when designing future e-Loran networks and determining their coverage and performance.
\end{abstract}

\section{Keywords:}

e-Loran, Cross-Rate Interference, signal processing. 


\section{INTRODUCTION}

The General Lighthouse Authorities of the United Kingdom and Ireland (GLAs) have recently produced a Business Case for e-Loran, selecting it as the most cost-effective way of providing resilient Positioning, Navigation and Timing (PNT) for maritime e-Navigation. The GLAs currently operate a trial e-Loran service from Harwich, UK and are working towards establishing e-Loran Initial Operational Capability (IOC) in the seven busiest UK ports and port approaches by mid-2013. After IOC, the GLAs will look to extend e-Loran across their entire service area. This extension of e-Loran coverage will require the installation of additional transmitting stations. When planning the installation of e-Loran transmitters service providers will need a good understanding of the effects of the new signals on the system's performance. Since all e-Loran stations share the same frequency band and the e-Loran signals propagate over vast distances, special attention needs to be paid to the issue of intra-system interference.

e-Loran is a time-division system. The transmitters are organised in groups of usually 3 to 5 stations called chains or rates. The stations periodically broadcast short groups of radio pulses at a given Group Repetition Interval (GRI). Careful selection of GRIs and transmission times ensures that stations operating in a chain do not interfere with each other. However, the nature of the system is such that pulses from different GRIs overlap for a certain percentage of time. This is usually referred to as Cross-Rate Interference (CRI) and, if left uncompensated, is a major source of measurement error in Loran systems.

The issue was recognised relatively early in the development of Loran systems and a number of methods were suggested to avoid excessive interference between Loran- $C$ chains (see e.g. [4, 7, 12]). However, due to the complicated nature of the interference and a lack of published information on e-Loran receivers, very little work has been done on modelling the effects of CRI on the system's performance.

The authors of this paper have previously addressed some aspects of the problem in $[10,11]$. The present paper follows from the authors' previous work and concentrates solely on the receiver end of the system. First, a model of the received e-Loran signal is created and a signal processing model for an e-Loran receiver is suggested. The paper goes on to present numerical and, where possible, analytical results describing the achievable accuracy performance under different noise and interference conditions. The theoretical results are compared to those obtained from measurements made on a commercially available receiver driven by an experimental signal simulator. Finally, some applications of the developed models are briefly discussed. 


\section{METHOD}

This section gives some detail on the signal model and the receiver signal processing model and their use in the subsequent performance analysis. Also included is a brief description of the signal simulator that was used to validate the performance models presented in this work.

\section{e-Loran Signal Model}

Each e-Loran station broadcasts groups of 8 low-frequency phase-coherent pulses with a given Group Repetition Interval (GRI), $T_{\mathrm{GRI}}$, and a nominal time offset ${ }^{1}$, $\tau=\tau_{\mathrm{ED}}$, as per Equation (2) below. The pulses can be described by the following expression ${ }^{2}$ :

$$
\tilde{a}\left(t ; \tau, \theta_{0}\right)=A e(t-\tau) \cos \left(2 \pi f_{\mathrm{c}} t+\theta_{0}\right),
$$

where $A$ is a normalisation constant, $e(t)$ defines the pulse envelope (see the original U.S. Coast Guard signal specification [13]), $t$ is time in seconds, and $f_{\mathrm{c}}=100 \mathrm{kHz}$ is the Loran carrier frequency. The pulse length is approximately $300 \mu \mathrm{s}$ and the time separation between the pulses in a group, $T_{p}$, is $1 \mathrm{~ms}$. The carrier phase of the pulses, $\theta_{0}$, is locked to a Caesium standard, however, the phase of some of the pulses is inverted according to special codes [13], $\mathcal{C}=\left\{C_{k}\right\}_{k=0}^{15}, C_{k}= \pm 1$, which enable automatic synchronisation of the receiver to the signals and help suppress some forms of interference. Two sets of codes are currently in use; these are referred to as the Master and the Secondary phase code. The phase code values repeat after a time interval of $2 T_{\mathrm{GRI}}$, also referred to as the Phase Code Interval (PCI).

$$
\begin{array}{r}
\tilde{s}\left(t ; T_{\mathrm{GRI}}, \tau_{\mathrm{ED}}, \theta_{0}, \mathcal{C}\right) \\
=\sum_{l=-\infty}^{\infty} \sum_{k=0}^{7}\left[C_{k} \tilde{a}\left(t-k T_{p}-2 l T_{\mathrm{GRI}} ; \tau_{\mathrm{ED}}, \theta_{0}\right)\right. \\
\left.+C_{k+8} \tilde{a}\left(t-k T_{p}-(2 l+1) T_{\mathrm{GRI}} ; \tau_{\mathrm{ED}}, \theta_{0}\right)\right]
\end{array}
$$

Equation (2) describes the basic e-Loran navigation signal. e-Loran also provides a data transmission capability. Several modulation techniques are known [3], designed in such a way that the navigation function is not significantly impaired. This paper concentrates on the positioning performance of e-Loran and the impact of possible data modulation is ignored in this analysis.

\footnotetext{
${ }^{1}$ Also called Emission Delay (ED).

${ }^{2}$ Throughout this paper, tilde denotes RF signals (as opposed to their complex envelopes).
} 
Equation (3) below shows the received signal model used in this analysis. The signal at the receiver antenna, $\tilde{x}(t)$, is modelled as a sum of signals from $N_{\mathrm{L}}$ e-Loran stations, contaminated by Continuous Wave Interference ${ }^{3}$ (CWI) and wideband RF noise, $\widetilde{w}_{\mathrm{RF}}(t)$. It is assumed that the CWI falls outside the Loran frequency band $\left(f_{n} \notin[90,110] \mathrm{kHz}\right.$; the Loran band is protected). For simplicity, the RF noise is modelled as white Gaussian process.

Each e-Loran signal in the model has a ground wave component and a sky wave component (only single-hop sky waves are modelled). The highly stable propagation characteristics of the ground wave transmissions make them ideally suited for navigation. The sky waves are essentially delayed copies of the signal arriving via reflection ${ }^{4}$ from the ionosphere, they are less predictable and generally considered a source of interference. The e-Loran signals may have different GRIs, potentially acting as sources of CRI. Sky wave borne CRI is of special importance, as the amplitude of the sky wave signal, $A_{m, s}$, is often significantly higher than that of the corresponding ground wave, $A_{m, \mathrm{~g}}$.

The useful information about the receiver's position is contained in the time offset, $\tau_{m, \mathrm{~g}}$, and carrier phase, $\theta_{m, \mathrm{~g}}$, of the ground wave signals.

$$
\begin{aligned}
& \tilde{x}(t)=\sum_{m=1}^{N_{\mathrm{L}}}[\underbrace{A_{m, \mathrm{~g}} \tilde{S}\left(t ; T_{\mathrm{GRI}, m}, \tau_{m, \mathrm{~g}}, \theta_{m, \mathrm{~g}}, \mathcal{C}_{m}\right)}_{\text {ground wave }} \\
& +\underbrace{A_{m, s} \tilde{s}\left(t ; T_{\mathrm{GRI}, m}, \tau_{m, \mathrm{~s}}, \theta_{m, \mathrm{~s}}, \mathcal{C}_{m}\right)}_{\text {sky wave }}] \\
& +\sum_{n=1}^{N_{\mathrm{CWI}}} \underbrace{B_{n} \cos \left(2 \pi f_{n} t+\theta_{n}\right)}_{\mathrm{CW} \text { interference }}+\underbrace{\widetilde{w}_{\mathrm{RF}}(t)}_{\mathrm{RF} \text { noise }}
\end{aligned}
$$

\section{Signal Processing Model for e-Loran}

The purpose of this section is to develop a signal processing model to allow the theoretical evaluation of e-Loran accuracy under different noise and interference conditions. Since e-Loran uses carrier phase positioning, the signal processing

\footnotetext{
${ }^{3} \mathrm{CWI}$ may originate from other radio services in the LF band or from local sources such as switched mode power supplies or computer displays.

${ }^{4}$ The term 'reflection' is usually used in connection with sky waves, although the phenomenon is in fact a combination of several complicated effects, and may be more accurately described as 'refraction'.
} 
model will essentially describe an algorithm to extract carrier-phase estimates of the ground wave signals, $\hat{\theta}_{m, \mathrm{~g}}$, from the composite received signal, $\tilde{x}(t)$. The relation between the carrier phase estimates, and the achievable positioning accuracy is discussed later in this paper.

The model assumes that: (i) the receiver is static (no attempts were made to evaluate the effects of the receiver dynamics on its performance); (ii) the receiver clock drift (frequency offset) has been compensated for (the receiver is frequencylocked to the e-Loran signal); (iii) feed-forward estimation is employed rather than a feed-back system (tracking loop) [6]. The complete signal processing model for a single channel of an e-Loran receiver is shown in figure 1 below. The following gives a brief description of the individual blocks of the proposed model. A rigorous derivation can be found in [8].

The first block in the suggested model is a bandpass filter. The filter limits the bandwidth of the RF noise entering the receiver, $\widetilde{w}_{\mathrm{RF}}(t)$, and suppresses all outof-band interference (CWI). This analysis assumed an 8 th order Butterworth filter with a $-3 \mathrm{~dB}$ bandwidth of $28 \mathrm{kHz}$ and the passband centred at $100 \mathrm{kHz}$. This appears to be a commonly accepted standard for modern e-Loran receivers [6].

For further processing and analysis it is convenient to convert the signals to baseband. This is a standard operation widely used in radio receivers (see e.g. [1]). At the output of the down-converter is the complex envelope, $x_{\mathrm{b}}(t)$, of the bandpass-filtered RF signal $\tilde{x}_{\mathrm{b}}(t)$. The magnitude of the complex envelope corresponds to the envelope of the original RF signal and its argument contains information about the signal's carrier phase - i.e. the quantity of interest.

As explained before, all e-Loran stations broadcast on the same carrier frequency, utilising a time-division scheme, where stations of different chains operate at different repetition rates. The receiver therefore needs a means of selecting signals of a particular rate (GRI) and suppressing those of other rates that may also be present. The natural way of achieving that is the averaging of the received signal over the repetition interval of the wanted station (recall that because of the phase codes, the idealised e-Loran signal is periodic in $2 T_{\mathrm{GRI}, m}$ ):

$$
x_{\mathrm{c}}(t)=\frac{1}{N_{\mathrm{i}}} \sum_{l=0}^{N_{\mathrm{i}}-1} x_{\mathrm{b}}\left(t-2 l T_{\mathrm{GRI}, m}\right),
$$

where $N_{\mathrm{i}}=T_{\mathrm{i}} /\left(2 T_{\mathrm{GRI}, m}\right)$, and $T_{\mathrm{i}}$ is the integration period, typically 5 seconds or so (for simplicity it is assumed throughout this paper that $T_{\mathrm{i}}$ is an integer multiple of $\left.2 T_{\mathrm{GRI}, m}\right)$. 
Equation (4) implies the use of a structure commonly called the comb filter. The name stems from the shape of the magnitude response of this filter. The filter accentuates spectral lines of signals with a given GRI and thus allows the user to 'tune' the receiver to a particular chain. It is the simplest CRI suppression technique, inherently present in all Loran receivers.

The next block is the phase decoding filter. This filter allows the receiver to identify individual signals within a chain and helps to suppress long delay ${ }^{5}$ sky waves and some other forms of interference [8]. It makes use of the correlation properties of the e-Loran phase codes, $\mathcal{C}=\left\{C_{k}\right\}_{k=0}^{15}$, and its operation can be described in the time domain as follows:

$$
\begin{aligned}
x_{\mathrm{p}}(t)=\frac{1}{16} \sum_{k=0}^{7} & {\left[C_{k} x_{\mathrm{c}}\left(t-2 T_{\mathrm{GRI}, m}+k T_{\mathrm{p}}\right)\right.} \\
& \left.+C_{k+8} x_{\mathrm{c}}\left(t-T_{\mathrm{GRI}, m}+k T_{\mathrm{p}}\right)\right] .
\end{aligned}
$$

Note that the combination of the phase-decoding filter and the comb filter effectively performs coherent averaging ${ }^{6}$ of pulses received within the past integration interval, $T_{\mathrm{i}}$.

To avoid own interference from short-delay ${ }^{7}$ sky waves, e-Loran receivers use for absolute positioning only the leading part of each received pulse. In the proposed signal processing model, this is done by sampling the averaged pulse at the output of the phase-decoding filter at time instants defined by

$$
t_{u}=u T_{\mathrm{up}}+\tau_{\mathrm{sp}}
$$

where $T_{\text {up }}$ is the update interval (typically 1 second, or same as $T_{\mathrm{i}}$, and again, it is assumed that $T_{\text {up }}$ is an integer multiple of $\left.2 T_{\mathrm{GRI}, m}\right), u \in \mathbb{Z}$ and $\tau_{\mathrm{sp}}$ is an appropriately chosen sampling point offset. The choice of the sampling point location is a compromise between low signal strength at the beginning of the pulse and higher probability of sky wave contamination closer to the pulse peak. The location may differ between receiver makes. At the output of the sampler is a discrete-time signal, here denoted as $x_{\mathrm{p}}[\mathrm{u}]=x_{\mathrm{p}}\left(t_{\mathrm{u}}\right)$.

The final block is the phase detector, which transforms the pulse samples $x_{\mathrm{p}}[\mathrm{u}]$ into carrier phase estimates, $\hat{\theta}_{m, \mathrm{~g}}$. This is easily achieved by calculating the argument of the (complex) pulse samples, $\hat{\theta}_{m, \mathrm{~g}}=\angle x_{\mathrm{p}}[\mathrm{u}]$.

\footnotetext{
${ }_{6}^{5}$ Sky waves delayed by approximately $700 \mu$ s or more.

${ }^{6}$ The averaging operation is sometimes referred to in literature as 'integration'.

${ }^{7}$ Sky waves delayed by approximately $35 \mu$ s to $700 \mu$ s.
} 
It can be shown [8] that the phase estimation algorithm described above implements the Maximum Likelihood (ML) carrier phase estimator for an Additive White Gaussian Noise (AWGN) channel (i.e. in the absence of CRI). Optimal reception in the presence of CRI requires some additional signal processing.

Cross-rating e-Loran signals are largely suppressed by the averaging applied in the comb and phase-decoding filters. However, as shown later in this paper, the suppression may not always be sufficient for the stringent e-Loran performance standards to be met. To further reduce the effects of CRI e-Loran receivers employ special CRI mitigation algorithms. These algorithms are represented in the suggested model by an additional signal processing block inserted between the downconverter and the main comb filter. Several CRI mitigation strategies have been described in the literature [5]. This paper deals with the two most important ones, commonly referred to as CRI blanking and CRI cancelling.

CRI blanking is a simple yet effective way of mitigating CRI through the detection and censoring of the pulses likely corrupted by the interference. Figure 2 (left) shows a signal processing model for this technique adopted in this study. The model assumes that the receiver is processing signals from both cross-rating stations so that the time when the pulse groups overlap can be predicted.

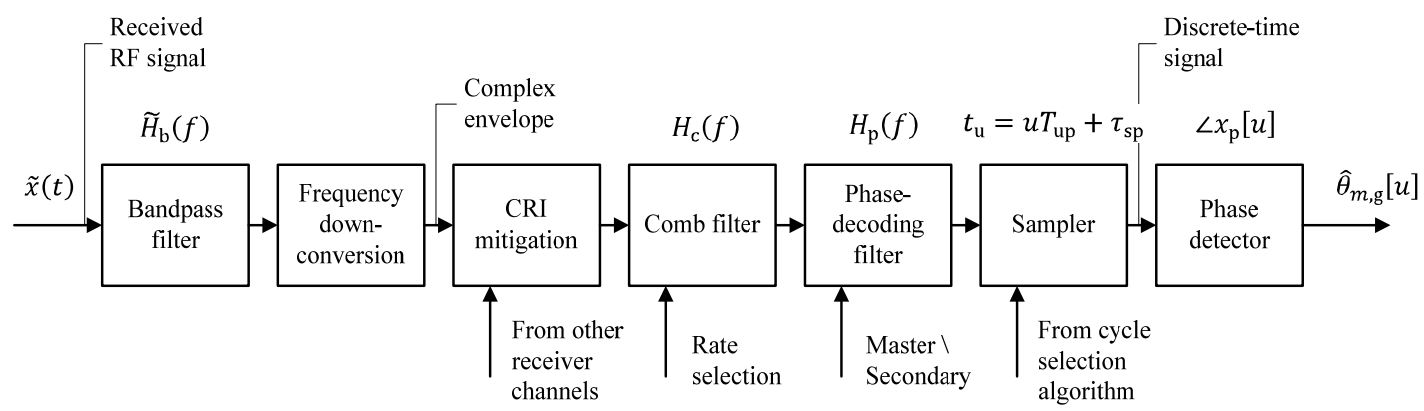

Fig. 1. Receiver signal processing model for e-Loran [own study]

By discarding all e-Loran pulses that overlap, it is possible to completely suppress the interference; however, the price paid is a (sometimes excessive) loss of useful signal energy. This in turn amplifies the errors due to background noise, as shown later in the paper.

Another possibility is the use of CRI cancelling. With this technique, the receiver reconstructs replica waveforms of the interference and subtracts them from the composite received signal, effectively cancelling the interference while leaving the useful signal unharmed. A signal processing model for this technique is shown in figure 2 (right). The model uses an additional comb filter to generate the replica of interference originating from a given GRI. 
Clearly, the effectiveness of the method depends on the accuracy of the replica signals. In practice, there are a number of reasons why the cancellation is never perfect. First, the replicas are obtained by averaging of the received, noisy, signals and are therefore contaminated by some residual noise (and interference). Also the timing and amplitude stability of the transmitted signals is an important factor. Pelgrum [5] states that the transmitted pulses can jitter by $50 \mathrm{~ns}$ in time and $2 \%$ in amplitude (understood as one sigma values) ${ }^{8}$. Since the cancelling algorithm works with averaged waveforms, the pulse-to-pulse fluctuations cannot be reproduced, and the effectiveness of the algorithm is reduced. The impact of pulse jitter on the performance of CRI cancelling is examined later in this paper. Next, during the averaging and cancelling operations, the receiver must compensate for the (generally unknown) clock drift and the receiver's motion, which poses a significant challenge (these effects are not investigated in this analysis). Further complications may arise when the cross-rating signal is data modulated. In that case, the digital information has to be recovered and re-modulated onto the replica signal (data channel considerations are out of the scope of this analysis).
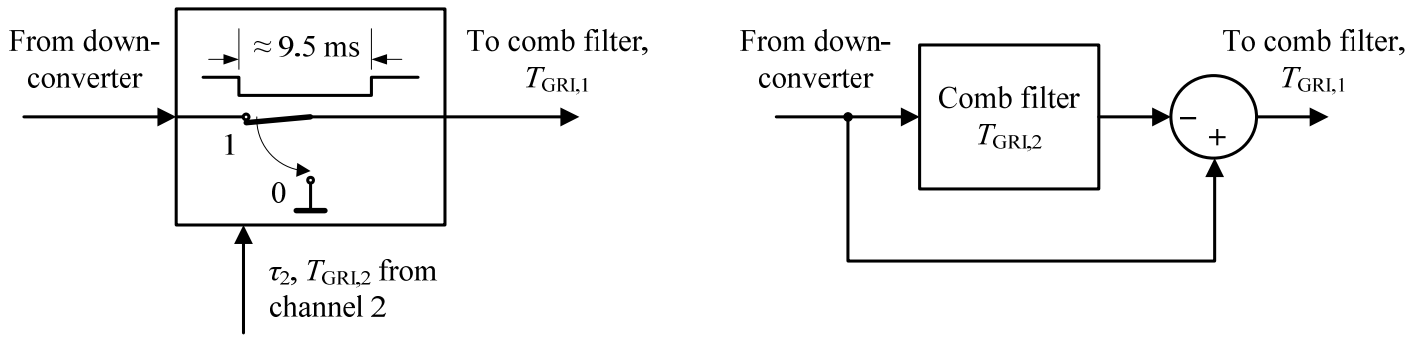

Fig. 2. Receiver signal processing model for CRI blanking (left) and CRI cancelling [own study]

\section{Performance Analysis}

The signal processing model outlined in this paper can be used to evaluate the phase measurement error under given noise and interference conditions:

$$
\varepsilon_{\theta}[u]=\hat{\theta}_{m, \mathrm{~g}}[u]-\theta_{m, \mathrm{~g}} .
$$

Assuming that the receiver correctly resolves the inherent ambiguity ${ }^{9}$ of the phase measurements, the phase measurement error, $\varepsilon_{\theta}[u]$, directly translates into a ranging error

\footnotetext{
${ }^{8}$ Although no figures can be provided, it is believed that modern eLoran transmitter equipment achieves somewhat better timing and amplitude stability.

${ }^{9}$ The ambiguity resolution is performed by the so called 'cycle selection' algorithm and is not dealt with in this paper. Note that failure to resolve the ambiguity results in ranging errors of $3 \mathrm{~km}$ or more and is therefore an integrity issue.
} 


$$
\varepsilon_{\mathrm{r}}[u]=c \frac{\varepsilon_{\theta}[u]}{2 \pi f_{\mathrm{c}}},
$$

where $c$ is the signal propagation velocity and $f_{\mathrm{c}}$ is as defined above.

Because of the periodic ${ }^{10}$ nature of CRI, it was decided to model the ranging error as a cyclostationary random process. Since the statistical properties of cyclostationary processes vary cyclically with time, the usual approach to dealing with such processes is through the use of average characteristics (i.e. both time- and ensable- averaged, $\operatorname{Av}[\mathrm{E}[\cdot]] \equiv \operatorname{AvE}[\cdot])$. The following performance metrics were therefore chosen:

The average ranging error (bias)

$$
\mu_{\varepsilon_{\mathrm{r}}}=\operatorname{AvE}\left[\varepsilon_{\mathrm{r}}[u]\right]
$$

and the average power of the ranging error

$$
P_{\varepsilon_{\mathrm{r}}}=\operatorname{AvE}\left[\left|\varepsilon_{\mathrm{r}}[u]\right|^{2}\right]=\operatorname{var}\left[\varepsilon_{\mathrm{r}}[u]\right]+\mu_{\varepsilon_{\mathrm{r}}}^{2} .
$$

It is assumed that the receiver uses the Weighted Least Squares (WLS) algorithm to calculate its position. The projection of the ranging errors into the position domain is then a standard exercise (see [11] or any good textbook on radionavigation).

The beauty of the presented model is in its simplicity. It can be linearized under reasonable assumptions, which often allows the derivation of analytical expressions for the error statistics. The model was also implemented in MATLAB $\backslash$ Simulink ${ }^{\circledR}$, which allows numerical simulations to be performed in cases where the analytical approach becomes intractable.

\section{Valid a tion}

In order to validate the signal processing and performance models presented in this paper, an experimental e-Loran signal simulator set-up has been developed through cooperation between the GLAs and CTU Prague. The simulator allows the creation of a controlled radio environment in the laboratory and enables performance testing of commercially available receivers under repeatable conditions.

The prototype set-up comprises a PC workstation equipped with a multichannel DA converter board, GPS-disciplined Rubidium clock and an antenna coupler. The workstation runs software written by the lead author, which allows the generation of e-Loran signals according to the signal model presented above. The software-defined architecture of the simulator allows an easy integration of new signal and noise sources. For more detail about the set-up see [10].

${ }^{10}$ The (idealised) eLoran signals are periodic and their periods are rational numbers, therefore the interfering patterns also have a periodic nature. 


\section{RESULTS}

\section{Ranging Error due to RF Noise}

The analysis first focused on ranging errors caused by RF noise, which limits the performance of the receiver in the absence of interference. In this case, the signal model consisted of a single ground-wave e-Loran signal embedded in White Gaussian Noise $\left(N_{\mathrm{L}}=1, A_{1, \mathrm{~s}}=0, N_{\mathrm{CWI}}=0\right.$ in Equation (3).

Using the signal and receiver models described above, it was shown [8] that the ranging error is inversely proportionate to the number of pulses in the integration period $^{11}, N_{\mathrm{p}}$, and the Signal-to-Noise Ratio, $S N R^{12}$ (expressed as a ratio rather than $\mathrm{dB}$ ):

$$
P_{\varepsilon_{\mathrm{r}}}=\operatorname{var}\left[\varepsilon_{\mathrm{r}}\right]=\frac{c^{2}}{8\left(\pi f_{\mathrm{c}}\right)^{2} N_{\mathrm{p}} \cdot S N R} \approx \frac{337.4^{2}}{N_{\mathrm{p}} \cdot S N R} \text {. }
$$

Equation (11) confirms earlier work done at Stanford University [2], and was also verified numerically and against simulator measurements. The measurement errors observed in the simulator experiments were somewhat higher than the theoretical predictions and the effect was calibrated out using an additional multiplicative factor:

$$
P_{\varepsilon_{\mathrm{r}}} \approx L_{\mathrm{impl}} \frac{337.4^{2}}{N_{\mathrm{p}} \cdot S N R} .
$$

The factor represents various implementation losses and uncertainties about the values of some of the receiver parameters (such as the exact location of the sampling point). For the specific receiver used in this study ${ }^{13} L_{\text {impl }} \approx 4.8$.

\section{Ranging Error due to CRI}

\section{CRI Suppression by Averaging}

The main focus of this work was on Cross-Rate Interference and its mitigation. The first part of this analysis investigated the achievable CRI suppression due to the averaging applied in the comb and phase-decoding filters. In this case, the signal model consisted of two cross-rating ground wave signals $\left(N_{\mathrm{L}}=2, T_{\mathrm{GRI}, 1} \neq T_{\mathrm{GRI}, 2}\right.$, $A_{1, \mathrm{~s}}=A_{2, \mathrm{~s}}=0, N_{\mathrm{CWI}}=0, \widetilde{w}_{\mathrm{RF}}(t)=0, \forall t$ in Equation (3). The signal processing model shown in figure 1 was used; the CRI mitigation block was omitted.

${ }^{11} N_{\mathrm{p}}=8 T_{\mathrm{i}} / T_{G R I}$, assuming 8 pulses per GRI.

${ }^{12}$ Due to the pulsed nature of the signal and different types of input filters in use, SNRs have traditionally been a matter of confusion in Loran systems; for exact definition of SNR used by the authors see [9].

${ }^{13}$ Reelektronika LORADD with Plutargus v1.0 firmware. 
A frequency-domain method has been devised that enables a rapid evaluation of the CRI-induced errors. The full description of the method falls out of the scope of this paper; for more detail the reader is referred to [8].

As an example of using the method, figure 3 below shows the RMS ranging error, $\sqrt{P_{\varepsilon_{\mathrm{r}}}}$, for a GRI 6731 signal cross-rating with a GRI 7001 signal. The results of this analysis show that uncompensated CRI can easily cause ranging errors on the order of meters. The errors are a complicated function of many parameters, such as the Signal-to-Interference Ratio, $S I R$, the time-alignment between the pulse trains, $\Delta \tau=\tau_{2, \mathrm{~g}}-\tau_{1, \mathrm{~g}}$, or the GRIs. Another noteworthy feature of uncompensated CRI, illustrated by figure 4 , is that the errors do not average out. There is a certain maximum integration time beyond which further averaging does not help. This is most likely due to the fact that the e-Loran phase codes are not balanced. This effect may be of particular importance in static monitoring receivers (e.g. for timing applications) where long integration periods are acceptable.

As shown in [8], the frequency-domain approach can also be extended to describe the effects of sky wave-borne CRI and multiple interferers.

The model is in very good agreement with simulator measurements for high SIR values, which suggests that weak interferers are left uncompensated by the specific receiver used in the test.

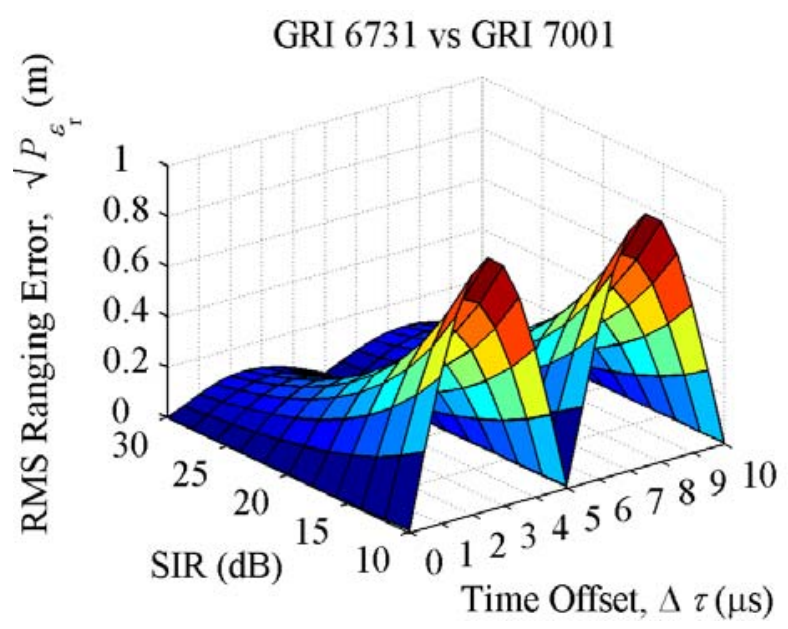

Fig. 3. Ranging error due to uncompensated CRI as a function of SIR and the time offset between signals [own study] 


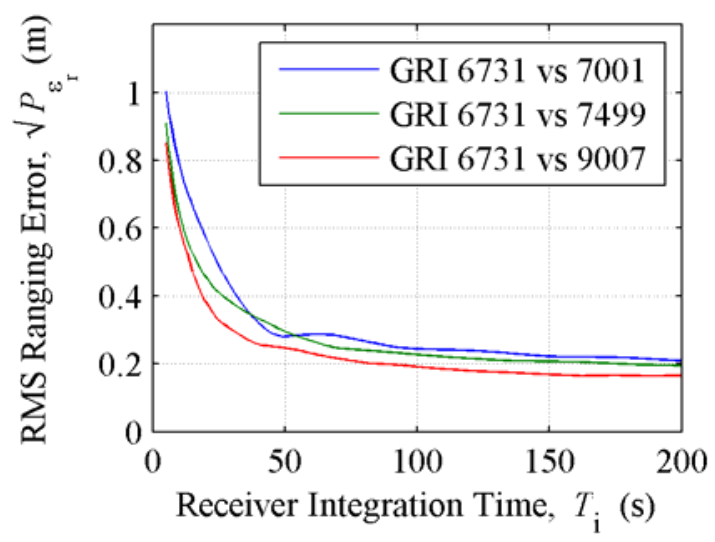

Fig. 4. Ranging error due to uncompensated CRI as a function of the receiver integration time; $\mathrm{SIR}=10 \mathrm{~dB}$, time offset between signals, $\Delta \tau=2.5 \mu \mathrm{s}$ [own study]

\section{CRI Mitigation by Blanking}

As discussed earlier in this paper, the errors caused by CRI can further be reduced through the use of special CRI mitigation algorithms. This paragraph focuses on the simplest of these techniques - CRI blanking.

CRI blanking completely eliminates the CRI-induced errors but the use of the technique may result in a considerable loss of useful signal energy. To demonstrate the effect on the ranging performance, the received signal in this part of the analysis was modelled as a sum of several cross-rating e-Loran signals embedded in White Gaussian Noise $\left(N_{L} \geq 2, T_{G R I, 1} \neq T_{G R I, m}, m>1, N_{\mathrm{CWI}}=0\right.$ in Equation (3).

The blanking loss ${ }^{14}$ due to multiple interferers, $L_{\mathrm{b}}$, was evaluated using methods presented in an earlier paper by the authors [10]. The ranging error was then estimated using Equation (12), where the number of averaged pulses, $N_{\mathrm{p}}$, was reduced accordingly, i.e. $\left(1-L_{\mathrm{b}}\right) N_{\mathrm{p}}$ was used instead of $N_{\mathrm{p}}$.

Figure 5 below illustrates the residual effect of CRI on a GRI 6731 signal embedded in WGN when cross-rating signals from other European GRIs are suppressed. The figure shows that when all cross-rating European stations are blanked, the ranging performance in WGN is approximately 2.3 times worse than if there was no CRI. Whether it is advantageous for the receiver to use blanking (and suffer some blanking loss) or not (and suffer some error due to uncompensated CRI) depends on the SIR and SNR.

The presented model for CRI blanking is in excellent agreement with simulator measurements for low SIR values.

\footnotetext{
${ }^{14}$ The percentage of wanted pulses lost as a result of mitigating the CRI through blanking.
} 


\section{CRI Mitigation by Cancelling}

This section presents the results of our investigation into the effectiveness of CRI cancelling. The signal model in this part of the analysis consisted of two cross-rating ground wave e-Loran signals $\left(N_{\mathrm{L}}=2, T_{G R I, 1} \neq T_{G R I, 2}, A_{1, \mathrm{~s}}=A_{2, \mathrm{~s}}=0, N_{\mathrm{CWI}}=0\right.$, $\widetilde{w}_{\mathrm{RF}}(t)=0, \forall t$ in Equation (3). In order to demonstrate some of the practical limitations of cancelling, the amplitude of the interfering signal ( $A$ in Equation (1) was jittered from pulse to pulse according to a normal distribution; in practice, some amplitude jitter is always present due to transmitter imperfections (see above) and changing propagation conditions.

Numerical simulations in MATLAB $\backslash$ Simulink ${ }^{\circledR}$ were performed to evaluate the ranging performance for different SIR values and different amounts of jitter in the interfering signal. The results of these simulations are shown in figure 6 below.

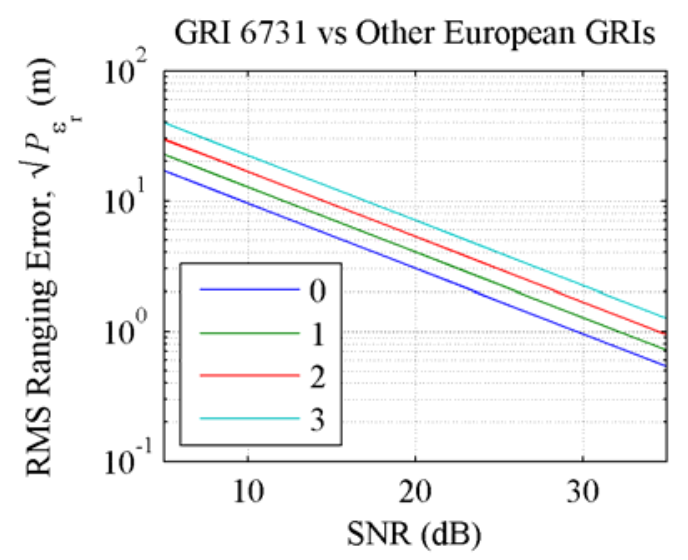

Fig. 5. Residual ranging error for a GRI 6731 signal interfering with other European GRIs after CRI blanking; parameter: number of interfering chains [own study]

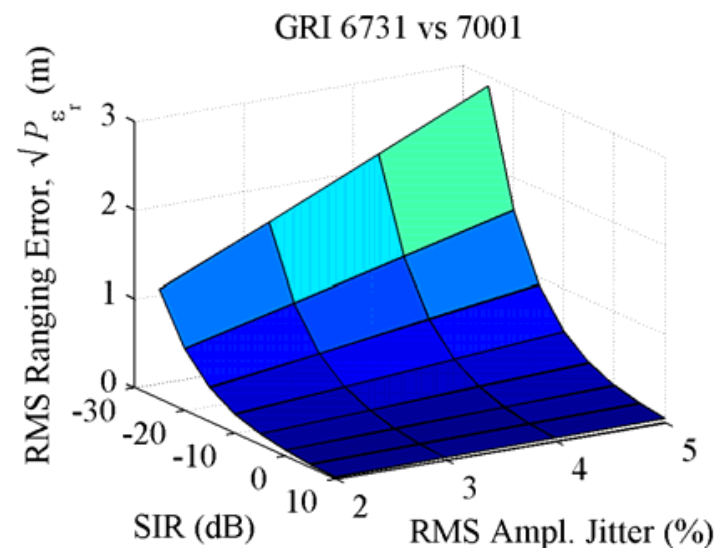

Fig. 6. Residual ranging error due to CRI after CRI cancelling [own study] 
As expected, the residual errors increase with decreasing SIR and increasing amount of jitter. The effect becomes noticeable at approximately $0 \mathrm{~dB}$ SIR; weaker interference is practically perfectly cancelled. The favoured strategy for CRI mitigation therefore seems to be the blanking of stronger interferers and cancelling of the weaker ones. Cross-rating signals that are at least $20 \mathrm{~dB}$ (or so) weaker than the wanted signal can safely be ignored (see fig. 3).

\section{DISCUSSION AND CONCLUSIONS}

Prior work has documented that CRI is a serious issue in Loran systems. However, due to the complex nature of the interference and a lack of published information on (e)Loran receivers, very little work has been done on CRI modelling. In this study a signal processing model for e-Loran was developed and the effects of CRI were analysed theoretically. It has been shown that the use of modern e-Loran signal processing algorithms can substantially reduce the impact of CRI. The theoretical results confirm and extend those of Pelgrum [5], allowing the quantification of the CRI-related errors under a variety of conditions. Performance predictions based on the models presented in this paper are also in a very good agreement with simulator measurements.

However, some limitations are worth noting. First, the radio noise in this analysis was modelled as White Gaussian Noise; in reality, most of the noise in the LF band comes from lightning discharges (atmospheric noise) and may be significantly non-Gaussian in nature. Future work should investigate the implications of this for the optimal receiver structure and the achievable accuracy performance. It is also suggested to explore the possibilities of sky-wave aided tracking, as outlined in [5]. These investigations should go hand in hand with further development of the signal simulator.

It is expected that the models and methods developed during this work will become useful as tools for optimal design of new e-Loran systems. As an example of how our results can be utilised in coverage and performance prediction ${ }^{15}$, figure 7 above shows predicted repeatable accuracy over North-West Europe based on the existing European Loran transmission network. The figure compares the achievable accuracy for a receiver that does not employ any CRI mitigation with the performance of a typical e-Loran receiver, which mitigates CRI through blanking. It can be seen that modern e-Loran signal processing considerably improves the positioning performance, especially in the vicinity of dual-rated transmitters, such as Lessay in Northern

\footnotetext{
${ }^{15}$ For more information on the GLAs coverage and performance model see [13].
} 
France or Sylt in Germany. The models can also help service providers to minimise the effects of CRI through the judicious choice of GRIs and optimal power allocations. Further, the signal simulator, originally developed to verify the validity of the presented models, also proved useful in testing of commercial e-Loran receivers, and there are plans for its use in the development of performance standards for marine receivers under RTCM Special Committee 127.
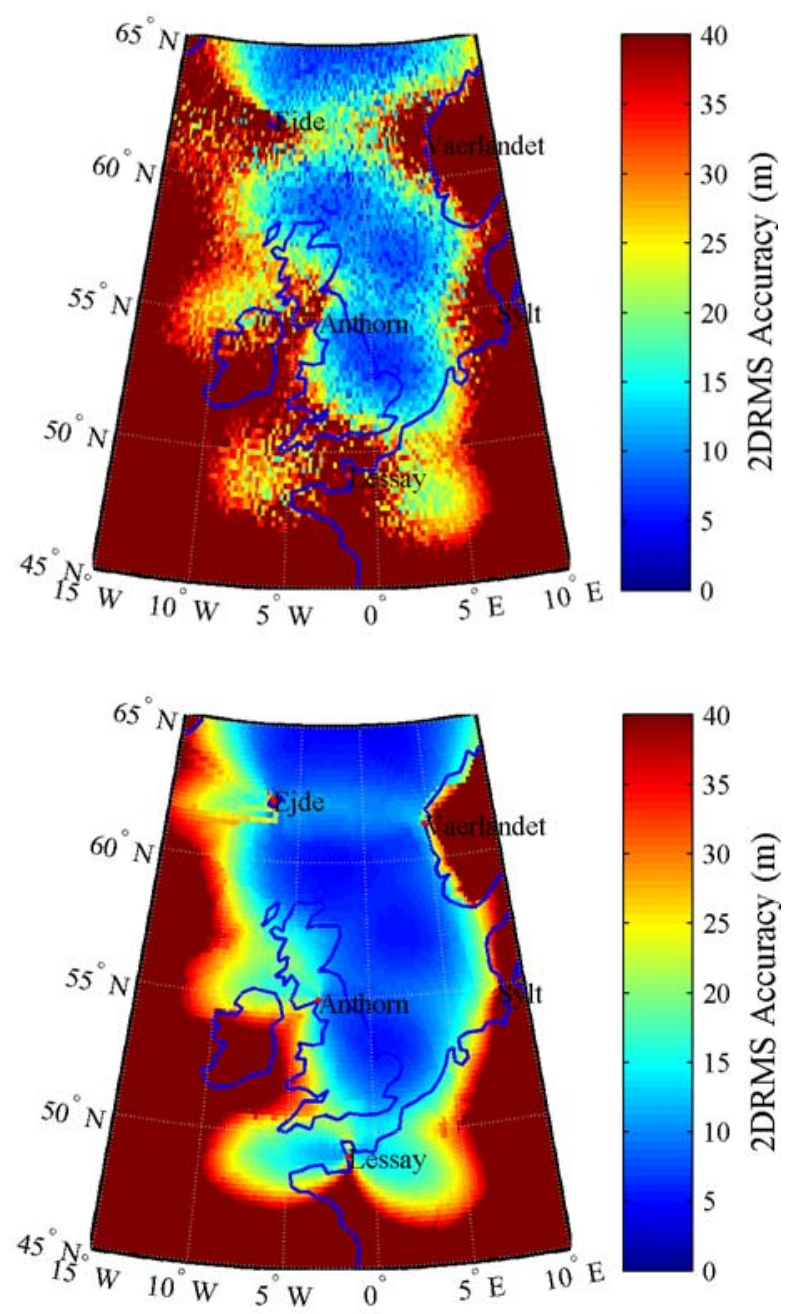

Fig. 7. Estimated positioning accuracy (2DRMS) assuming cross-rating ground wave signals only (day-time plot); performance without the use of CRI mitigation (left) vs. performance of a receiver employing CRI blanking; (based on work in progress) [own study] 


\section{REFERENCES}

[1] Haykin S., Communication Systems, $4^{\text {th }}$ ed. John Wiley and Sons, Inc., 2001.

[2] Lo S., Peterson B., Boyce C., Enge P., Loran Coverage Availability Simulation Tool, in Proceedings of the Royal Institute of Navigation (RIN) NAV08/ International Loran Association $37^{\text {th }}$ Annual Meeting, 2008.

[3] Lo S., Peterson B., Enge P., Loran data modulation: A primer, IEEE Aerospace and Electronic Systems Magazine, 2007, Vol. 22, No. 9, pp. 31-51.

[4] Nieuwland A. K., GRI Ranking Based on Cross-Rate Interference in Loran-C, The Journal of Navigation, 1995, Vol. 48, pp. 136-152.

[5] Pelgrum W., New Potential of Low-Frequency Radionavigation in the $21^{\text {st }}$ Century, Ph.D. dissertation, TU Delft, 2006.

[6] Personal discussions with Dr. Arthur Helwig (Reelektronika b.v.).

[7] Roland W. F., Loran-C Phase Code and Rate Manipulation for Reduced Cross Chain Interference, in Proceedings of the $3^{\text {rd }}$ Technical Symposium of the Wild Goose Association, 1974.

[8] Safar J., Cross-Rate Interference in e-Loran: Analysis and Mitigation, Ph.D. dissertation, Czech Technical University (CTU), Prague, to appear.

[9] Safar J., Lebekwe C. K., Williams P., Accuracy Performance of e-Loran for Maritime Applications, Annual of Navigation, 2010, Vol. 16, pp. 109-122.

[10] Safar J., Vejrazka F., Williams P., Assessing the limits of e-Loran positioning accuracy, in Proceedings of the TransNav 2011 International Symposium on Marine Navigation and Safety of Sea Transportation, A. Weintrit, ed., Gdynia Maritime University, Poland: CRC Press/Balkema, June 2011, pp. 55-63.

[11] Safar J., Williams P., Basker S., Vejrazka F., Group Repetition Interval Selection and Core e-Loran Service Capacity, in Proceedings of the $13^{\text {th }}$ IAIN World Congress, Stockholm 2009.

[12] Safar J., Williams P., Gug S., Group Repetition Interval Selection for e-Loran, in Proceedings of the Royal Institute of Navigation (RIN) NAV08/ International Loran Association (ILA) $37^{\text {th }}$ Annual Meeting, London, UK, 2008.

[13] Specification of the Transmitted LORAN-C Signal, United States Coast Guard, 1994, COMDTINST M16562.4A.

Received May 2012

Reviewed September 2012 